\title{
The Potential Influence of Absence of Blinding Method: Secondary Analysis From Randomized Controlled Trials Concerned Acupuncture for Depression
}

\section{Rui-Ting Wang}

Beijing University of Chinese Medicine

Ling-Jun Cui

Beijing University of Chinese Medicine

Jian-Ping Liu

Beijing University of Chinese Medicine

Mei Han

Beijing University of Chinese Medicine

Yu-Tong Fei

Beijing University of Chinese Medicine

Huijuan Cao ( $\nabla$ huijuancao327@hotmail.com )

Centre for Evidence-Based Chinese Medicine, Beijing University of Chinese Medicine, Beijing, China, 100029 https://orcid.org/0000-0003-3244-6288

\section{Research}

Keywords: Blinding method, performance bias, detection bias, randomized controlled trials, depression, acupuncture

Posted Date: September 24th, 2020

DOl: https://doi.org/10.21203/rs.3.rs-80123/v1

License: (1) (1) This work is licensed under a Creative Commons Attribution 4.0 International License.

Read Full License 


\section{Abstract}

\section{Background}

To evaluate the possibility and impact of performance and detection bias in randomized controlled trials due to lack of the blinding method.

\section{Methods}

Trials assessing acupuncture for depression published by March 2020 were searched from China National Knowledge Infrastructure database. Through matching, we reassembled the trials of comparing experimental acupuncture and control acupuncture. Meta-analysis with post-treatment HAMD (Hamilton Depression Scale) of the newly reassembled trials was conducted. Changes of HAMD of all included groups were also analyzed, the between groups difference was then analyzed by $t$-test. All statistical analyses were performed using RevMan 5.3 software.

\section{Results}

Thirteen pairs of studies were matched from 63 included trials. Re-analysis from the paired studies showed obvious difference of HAMD scales between the experimental and control acupuncture (Mean Difference was -2.95 for HAMD-17, -5.55 for HAMD-24). Re-analysis from all the included groups also showed the statistical difference between groups at four weeks of HAMD-17 ( $P=0.01)$, and four/six weeks of HAMD-24 $(P<0.01)$.

\section{Conclusion}

Effectiveness of acupuncture for decreasing the severity of depression was superior in trials that considered acupuncture as experimental treatment, showing performance and detection bias caused by the absence of blinding method is of great significance in acupuncture trials.

\section{Background}

The uncertainty principle attaches great significance to the uncertain state of therapeutic effects of the methods used in clinical trials, which is essential for controlling bias. A case in point is a study by Benjamin Djulbegovic discovered that clinical trials sponsored by the pharmaceutical industry result in biased findings, either due to selective report of studies with non-equivalent arms or publication of lowquality papers, wherein unfavorable results are incompletely described [1].

However, it is difficult for researchers to eliminate the participants' preference of intervention effect. Therefore, in order to avoid the influence of subjective will or preference on the research results, the study design principle came into being. Among these principles, blinding method is widely used in randomized controlled trials to avoid performance bias and detection bias [2]. The premise of success in implementing blinding method is that the experimental intervention has a reasonable placebo control, which is almost 
impossible for most non-pharmaceutical therapies, such as acupuncture. Thus, in case of absence of blinding, the researcher's values or preference may affect the procedure of the study and the results [3]. Study by Lingling Yang [4] categorizes several clinical trials according to therapy and grouping, and whether acupuncture has significant effect varied remarkably.

For centuries, literatures published about traditional Chinese medicine have been all about personal experiences. Although the rise of clinical epidemiology and evidence-based medicine has greatly improved researchers' understanding of the rigor of research design, trials' methodological quality has not improved substantially [5]. A case in point is a meta-analysis study by Mike Armour suggested that [6] 'A test for between acupuncture and control group differences showed that trials from China had significantly greater reductions in the severity of depression compared to those undertaken outside of China (Mean Difference $=0.58,95 \% \mathrm{Cl} 0.25$ to $0.91, \mathrm{P}<0.001$ ). This may be caused by selection and reports of favored studies or other bias originated from non-equivalent control.

Performance bias and detection bias, originated from researchers' preference of a certain therapy and insufficient use of the blinding method. To evaluate whether and how these two biases may affect the results of acupuncture clinical trials, we re-analyzed the data from the eligible acupuncture trials through meta-analysis method.

\section{Methods}

\section{Eligibility criteria}

Published randomized controlled trials with completely reported continuous data were concerned to be included. "Completely reported" means that mean of the outcome measurements for each group must be reported with their standard deviation (SD) or 95\% confidence interval (Cl) The target diseases are primary or secondary depression diagnosed in accordance with recognized criteria (e.g. Diagnostic and Statistical Manual of Mental Disorders Fifth Edition).

Acupuncture is the intervention of this study. By comparing the groups that were designed as experimental treatment and as control, we were able to explore whether the researchers' expectations will affect the results of acupuncture therapy for depression. Trials which employed acupuncture as experimental treatment may choose no treatment or standard treatment as control, thus we filtered out the trials which compared acupuncture to no treatment or western drugs (such as fluoxetine hydrochloride capsules, etc.). In this case, acupuncture was considered as "experimental treatment" and this arm of the trial was counted as intervention/experiment group in our review. Trials which used acupuncture as control treatment were mostly aimed to investigate the effect of another non-pharmaceutical therapy (e.g. Cognitive therapy, psychotherapy, music therapy. Etc.) for depression, or to verify the effect of a special acupuncture (special manipulation or needle type) for the disease. In this case, the routine acupuncture group was identified as control arm in our review. 
Primary outcome of this study was the score of Hamilton Depression Scale (HAMD). Literatures with inaccessible data, as well as the piratical documents would be excluded.

\section{Searching strategy}

The Chinese National Knowledge Infrastructure Databases was searched, including the unpublished dissertations. The searching items included 'Acupuncture' AND 'Depression' in the scope of Title/Abstract, and "Random" in full text.

\section{Study screening and data exaction}

Two reviewers (RTW and LJC) independently screened the literature to identify trials that potentially meet the above inclusion criteria. Any disagreement was resolved through discussion with a third reviewer (HJC). A predesigned form was used to extract data from the included trials. Extracted information included: i) General information, including document number, title, first author, year(s) conducted, location (city, country), source, etc. ii) Participants information, including diagnostic criteria, inclusion criteria, exclusion criteria, source, sample size, age, gender, disease course. iii) Treatment information, such as the treatment principle, types of acupuncture, information of the acupuncturist, treatment duration, points selection, treatment frequency, co-intervention, etc. iv) Outcome measures, including the type of HAMD scales (17-scores or 24-scores), changes in HAMD score, post-treatment HAMD scores, time of the measurement.

\section{Data analysis}

All statistical analyses were performed using RevMan 5.3 (The Cochrane Collaboration) software.

Since data of "experimental acupuncture" and "control acupuncture" to be compared in our study were get from different trials, we tried to make the two acupuncture groups comparable by matching them. After completing the data exaction, we selected the matching experimental and control acupuncture groups to reassemble trials. The first principle of matching is that the two groups of patients have the same type of depression, type of HAMD scale, and the treatment frequency. Besides, there should be no statistical difference $(P>0.05)$ between two groups in baseline information, including age, sex ratio, and the pretreatment HAMD scores. The prescriptions of the acupoints were also matched, however, we could only guarantee the equality of the main treatment principle between the reassembled groups since most of the trials were with individualized points selection. We then re-analyzed the difference of post-treatment HAMD scores in the new paired-study through RevMan 5.3. A meta-analysis with mean difference (MD) and $95 \% \mathrm{Cl}$ was used to present the results. I2 value reflect the statistical heterogeneity among trials. Data from the included groups which failed to be matched were not included in this analysis.

Furthermore, we also analyzed data from all the included groups regarding to the changes of HAMD score pre and post treatment within groups. Then, we separately pooled the data from experimental acupuncture group and control acupuncture group with subgroup meta-analysis. Subgroup was classified according to the type of HAMD scale and treatment duration. Difference of the pooling result between groups was then analyzed by t-test. 


\section{Results}

\section{Study selection}

Totally 1659 clinical trials were retrieved through preliminary searching. After reading the title and abstract, 869 trials were screened out. And further another 700 trials were excluded after reading the full text. Sixty-three randomized controlled trials [7-69] were finally assessed to be eligible (Fig. 1).

All the included trials were published in Chinese. The sample size ranged from 20 to 212 . All cases were recruited from the outpatient/inpatient Department. Primary depression was concerned in 46 trials [7-26, $34-50,55,57-60,64-67]$. The remaining 17 trials targeted on secondary depression, including eight poststroke depression $[27,51-54,61-63]$, five premenopausal depression [28-31, 56, 69], two postpartum depression [32, 33], one Internet addiction depression [68].

Treatment principle of most of the included trials were regulating spirit and relieving depression, and the most frequently used five acupoints were PC6 (37 studies) $[9,11,12,14,16-18,20-23,25,31,34-37$, $39-40,42-45,47,49-52,54-58,61,63-64,68]$, DU20 (31 studies) [11, 12, 16-22, 25, 27, 29-31, 33-37, $40,46,51,52,55,56,58,60,62,64,66,68]$, LR3 (30 studies) [7, 14, 17, 19, 20, 22, 24, 25, 29, 30, 34, 36, 37, $40,42,44,46,47,49,50,54-59,64,66-68]$, HT7 (23 studies) $[12,13,17,19,20,23,25,34,40,42-46,49-$ $52,55-58,64]$, DU29 (20 studies) [16, 18, 19, 22, 25, 29-31, 34-37, 46, 51, 52, 55, 56, 60, 64, 66]. Thirty included studies used acupuncture as intervention [7-36], 29 of them compared acupuncture with western drugs [7-35] and the remaining trial compared the acupuncture with no treatment [36]. Thirty-three included studies used routine acupuncture as control [37-69], and the interventions of them included special acupuncture [37-60], western medicine plus acupuncture [61, 62], acupuncture plus moxibustion $[63,64]$, moxibustion [65, 66], cognitive therapy plus acupuncture [67], cognitive therapy [68], and acupoint catgut embedding [69]. Acupuncture with electronic stimulation is adopted in five trials' acupuncture group, four of them in experimental group [14, 21, 24, 26], one in control group [65].

Thirteen trials reported the HAMD-17 in four weeks [7, 8, 28, 29, 32, 37-39, 53, 60, 61, 64, 69], 11 trials reported HAMD-17 in six weeks [9-12, 35, 36, 40, 41, 62, 65, 68], one trial reported HAMD-17 in both four and six weeks [13] and 1 trial reported HAMD-17 in both four and eight weeks [14], one trial reported HAMD-17 in twelve weeks [31]. Seventeen trials reported HAMD-24 in four weeks [15-20, 27, 30, 33, 34, 42, $43,50-52,54,58]$. Two trials reported HAMD-24 in four weeks and twelve weeks [49, 66], one trial reported HAMD-24 in eight weeks [56]. Eleven trials reported HAMD-24 in six weeks [22-26, 44, 47, 48, 55, 57, 59]. Five trials reported HAMD-24 in both four and six weeks $[21,45,46,63,67]$.

\section{Re-analysis from the paired studies}

We have successfully got 13 pairs of studies, in which five pairs of them reported HAMD-17 scales $[9,13$, $14,31,36-38,41,65,69]$, and the remaining studies reported HAMD-24 scales $[15,18,20,22,23,25,27$, $30,43,44,46,56,57,63,66,67]$. The specific information of the paired-studies were listed in Table 1. Meta-analysis shows that the difference of HAMD-17 scales (MD=-2.95, 95\% Cl -4.75 to $-1.16,12=74 \%, 10$ trials, 298 participants, $\mathrm{P}=0.001)$ and HAMD-24 scales $(\mathrm{MD}=-5.55,95 \% \mathrm{Cl}-6.69$ to $-4.41, \mathrm{I} 2=38 \%, 16$ trials, 
440 participants, $\mathrm{P}<0.00001$ ) between the paired experimental and control acupuncture were both statistically significant (Fig. 2). The obvious statistical heterogeneity among the paired studies were caused by the difference of acupoints selection. 
Table 1

Characteristics of 13-paired included studies

\begin{tabular}{|c|c|c|c|c|c|c|c|}
\hline \multirow{2}{*}{$\begin{array}{l}\text { Pair of } \\
\text { the } \\
\text { study } \\
\text { (Study } \\
\text { ID) }\end{array}$} & \multirow{2}{*}{$\begin{array}{l}\text { Sample } \\
\text { size } \\
\text { (M/F) }\end{array}$} & \multirow{2}{*}{$\begin{array}{l}\text { Age } \\
\text { (Mean } \pm \\
\text { SD) }\end{array}$} & \multirow[t]{2}{*}{$\begin{array}{l}\text { Type of } \\
\text { depression }\end{array}$} & \multirow[t]{2}{*}{ Acupoint } & \multirow[t]{2}{*}{ Outcome } & \multicolumn{2}{|c|}{$\begin{array}{l}\text { HAMD scores (Mean } \\
\pm \text { SD) }\end{array}$} \\
\hline & & & & & & Baseline & $\begin{array}{l}\text { Post- } \\
\text { treatment }\end{array}$ \\
\hline \multirow{2}{*}{$\begin{array}{l}\text { E: Liu } \\
\text { P 2009 } \\
\text { [9] } \\
\text { C: } \\
\text { Wang } \\
\text { XN } \\
2017 \\
{[41]}\end{array}$} & \multirow{2}{*}{$\begin{array}{l}6 / 14 \\
10 / 13\end{array}$} & \multirow{2}{*}{$\begin{array}{l}47.35 \pm \\
7.5 \\
46.57 \pm \\
7.46\end{array}$} & \multirow[t]{2}{*}{ Primary } & \multirow{2}{*}{$\begin{array}{l}\text { GV20, PC6 } \\
\text { GV20, EX- } \\
\text { HN3, EX-HN1, } \\
\text { ST36, EX- } \\
\text { HN5, SP6 }\end{array}$} & \multirow{2}{*}{$\begin{array}{l}\text { HAMD-17 } \\
6 \text { weeks }\end{array}$} & $\begin{array}{l}19.6 \pm \\
2.21\end{array}$ & $\begin{array}{l}7.65 \pm \\
3.52\end{array}$ \\
\hline & & & & & & $\begin{array}{l}20.87 \pm \\
2.01\end{array}$ & $\begin{array}{l}13.48 \pm \\
2.99\end{array}$ \\
\hline \multirow{3}{*}{$\begin{array}{l}\text { E: } \\
\text { Zhou X } \\
2011 \\
{[13]} \\
\text { C: } \\
\text { Cheng } \\
\text { XF } \\
2017 \\
{[38]}\end{array}$} & $12 / 20$ & $\begin{array}{l}40.5(22- \\
59)\end{array}$ & \multirow[t]{3}{*}{ Primary } & \multirow{3}{*}{$\begin{array}{l}\text { BL15, BL18, } \\
\text { BL20, HT7, } \\
\text { ST40 } \\
\text { RN12, ST25, } \\
\text { ST37 }\end{array}$} & HAMD-17 & \multirow{3}{*}{$\begin{array}{l}24.72 \pm \\
7.1 \\
25.8 \pm \\
10\end{array}$} & \multirow{3}{*}{$\begin{array}{l}15.2 \pm 7.2 \\
20.7 \pm \\
10.3\end{array}$} \\
\hline & $14 / 16$ & $19-53$ & & & 4 weeks & & \\
\hline & & & & & & & \\
\hline \multirow{2}{*}{$\begin{array}{l}\text { E: } \\
\text { Wang } \\
\text { XF } \\
2007 \\
{[14]}\end{array}$} & $12 / 18$ & $18-68$ & \multirow[t]{3}{*}{ Primary } & \multirow{3}{*}{$\begin{array}{l}\text { GB12, LR3, } \\
\text { with } \\
\text { electronic } \\
\text { stimulation } \\
\text { BL15, BL18, } \\
\text { BL20, PC6, } \\
\text { ST36, SP6, } \\
\text { with } \\
\text { electronic } \\
\text { stimulation }\end{array}$} & HAMD-17 & \multirow{3}{*}{$\begin{array}{l}26.39 \pm \\
6.19 \\
24.31 \pm \\
5.03\end{array}$} & \multirow{2}{*}{$\begin{array}{l}7.26 \pm \\
1.26\end{array}$} \\
\hline & \multirow[t]{2}{*}{$8 / 22$} & $\begin{array}{l}37.41 \rrbracket \\
12.75\end{array}$ & & & \multirow[t]{2}{*}{$6 / 8$ weeks } & & \\
\hline $\begin{array}{l}\text { C: } \\
\text { Song } \\
\mathrm{MQ} \\
2006 \\
{[65]}\end{array}$ & & & & & & & \\
\hline \multirow{2}{*}{$\begin{array}{l}\text { E: Li } \\
\text { HB } \\
2015 \\
{[31]}\end{array}$} & \multirow{2}{*}{$\begin{array}{l}32 \\
42\end{array}$} & $\begin{array}{l}50.59 \pm \\
294\end{array}$ & \multirow[t]{3}{*}{ Secondary } & \multirow{2}{*}{$\begin{array}{l}\text { BL23, BL18, } \\
\text { BL15, DU20, } \\
\text { EX-HN1, } \\
\text { DU24, DU29, } \\
\text { PC6 }\end{array}$} & \multirow{2}{*}{$\begin{array}{l}\text { HAMD-24 } \\
\text { 12/4weeks }\end{array}$} & $\begin{array}{l}20.69 \pm \\
1.77\end{array}$ & $\begin{array}{l}9.14 \pm \\
4.78\end{array}$ \\
\hline & & $\begin{array}{l}48.5 \pm \\
4.20\end{array}$ & & & & $\begin{array}{l}22.1 \pm \\
4.34\end{array}$ & $\begin{array}{l}10.83 \pm \\
3.91\end{array}$ \\
\hline $\begin{array}{l}\text { C: Jin } \\
\text { YP } \\
2013 \\
\text { [69] }\end{array}$ & & & & $\begin{array}{l}\text { BL23, BL15, } \\
\text { BL18, BL20, } \\
\text { SP6 }\end{array}$ & & & \\
\hline
\end{tabular}

HAMD: Hamilton Depression Scale; M: Male; F: Female; T: Treatment; C: Control; SD: Standard Deviation; Cl: confidence level. 


\begin{tabular}{|c|c|c|c|c|c|c|c|}
\hline \multirow{2}{*}{$\begin{array}{l}\text { Pair of } \\
\text { the } \\
\text { study } \\
\text { (Study } \\
\text { ID) }\end{array}$} & \multirow{2}{*}{$\begin{array}{l}\text { Sample } \\
\text { size } \\
\text { (M/F) }\end{array}$} & \multirow{2}{*}{$\begin{array}{l}\text { Age } \\
\text { (Mean } \pm \\
\text { SD) }\end{array}$} & \multirow[t]{2}{*}{$\begin{array}{l}\text { Type of } \\
\text { depression }\end{array}$} & \multirow[t]{2}{*}{ Acupoint } & \multirow[t]{2}{*}{ Outcome } & \multicolumn{2}{|c|}{$\begin{array}{l}\text { HAMD scores (Mean } \\
\pm \text { SD) }\end{array}$} \\
\hline & & & & & & Baseline & $\begin{array}{l}\text { Post- } \\
\text { treatment }\end{array}$ \\
\hline \multirow{2}{*}{$\begin{array}{l}\text { E: Xie } \\
\text { M } \\
2018 \\
{[36]} \\
\text { C: } \\
\text { Chen } \\
\text { YY } \\
2011 \\
{[37]}\end{array}$} & \multirow{2}{*}{$\begin{array}{l}8 / 22 \\
13 / 18\end{array}$} & \multirow{2}{*}{$\begin{array}{l}42.13 \pm \\
13.11 \\
35.23 \rrbracket \\
12.5\end{array}$} & \multirow[t]{2}{*}{ Primary } & \multirow{2}{*}{$\begin{array}{l}\text { DU29, DU20, } \\
\text { PC6, SP6, } \\
\text { LR3 } \\
\text { DU20, DU29, } \\
\text { LR3, PC6 }\end{array}$} & \multirow{2}{*}{$\begin{array}{l}\text { HAMD-17 } \\
6 \text { weeks }\end{array}$} & \multirow{2}{*}{$\begin{array}{l}12.63 \pm \\
2.91 \\
13.8 \pm \\
2.32\end{array}$} & \multirow{2}{*}{$\begin{array}{l}6.6 \pm 3.96 \\
8.32 \pm \\
2.61\end{array}$} \\
\hline & & & & & & & \\
\hline \multirow{3}{*}{$\begin{array}{l}\text { E: } \\
\text { Chen } \\
\text { CW } \\
2007 \\
{[15]} \\
\text { C: } \\
\text { Chen L } \\
2015 \\
{[45]}\end{array}$} & \multirow{3}{*}{$\begin{array}{l}11 / 19 \\
18 / 11\end{array}$} & \multirow{3}{*}{$\begin{array}{l}40.45 \pm \\
12.54 \\
45.80 \pm \\
8.40\end{array}$} & \multirow[t]{3}{*}{ Primary } & \multirow{3}{*}{$\begin{array}{l}\text { GV20, GV16, } \\
\text { EX-HN3, EX- } \\
\text { HN1, BL18 } \\
\text { GV20, CV17, } \\
\text { PC6, HT7 }\end{array}$} & \multirow{3}{*}{$\begin{array}{l}\text { HAMD-24 } \\
4 \text { weeks }\end{array}$} & \multirow{3}{*}{$\begin{array}{l}26.6 \pm \\
6.26 \\
28.86 \pm \\
2.76\end{array}$} & \multirow{3}{*}{$\begin{array}{l}15.31 \pm \\
4.08 \\
\\
21.24 \pm \\
0.95\end{array}$} \\
\hline & & & & & & & \\
\hline & & & & & & & \\
\hline \multirow{2}{*}{$\begin{array}{l}\text { E: } \\
\text { Wang } \\
\text { C 2007 } \\
{[18]}\end{array}$} & $14 / 16$ & \multirow{2}{*}{$\begin{array}{l}39.73 \pm \\
13.38 \\
\\
38.26 \pm \\
11.8\end{array}$} & \multirow[t]{2}{*}{ Primary } & \multirow{2}{*}{$\begin{array}{l}\text { PC6, SJ5, } \\
\text { DU20, DU229 } \\
\text { LR3, LI4, } \\
\text { DU20, DU29 }\end{array}$} & \multirow{2}{*}{$\begin{array}{l}\text { HAMD-24 } \\
12 \text { weeks }\end{array}$} & \multirow{2}{*}{$\begin{array}{l}30.7 \pm \\
3.4 \\
29.15 \pm \\
3.61\end{array}$} & \multirow{2}{*}{$\begin{array}{l}13.04 \pm \\
7.87 \\
18.45 \pm \\
5.94\end{array}$} \\
\hline & 16/17 & & & & & & \\
\hline \multirow{3}{*}{$\begin{array}{l}\text { E: } \\
\text { Wang } \\
\text { QS } \\
2016 \\
{[20]} \\
\text { C: Yao } \\
\text { XY } \\
2016 \\
{[48]}\end{array}$} & $15 / 17$ & \multirow{3}{*}{$\begin{array}{l}41.3 \pm \\
5.2 \\
37.40 \pm \\
8.73\end{array}$} & \multirow[t]{3}{*}{ Primary } & \multirow{3}{*}{$\begin{array}{l}\text { DU20, SP6, } \\
\text { KI3, HT7, PC7, } \\
\text { LR3 } \\
\text { GV20, EX- } \\
\text { HN1, EX-HN5, } \\
\text { EX-HN3, LI4 }\end{array}$} & \multirow{3}{*}{$\begin{array}{l}\text { HAMD-24 } \\
8 / 6 \text { weeks }\end{array}$} & \multirow{3}{*}{$\begin{array}{l}23.65 \pm \\
2.12 \\
23.6 \pm \\
2.86\end{array}$} & \multirow{3}{*}{$\begin{array}{l}13.74 \pm \\
2.85 \\
17.3 \pm 4\end{array}$} \\
\hline & $4 / 6$ & & & & & & \\
\hline & & & & & & & \\
\hline
\end{tabular}

HAMD: Hamilton Depression Scale; M: Male; F: Female; T: Treatment; C: Control; SD: Standard Deviation; Cl: confidence level. 


\begin{tabular}{|c|c|c|c|c|c|c|c|}
\hline \multirow{2}{*}{$\begin{array}{l}\text { Pair of } \\
\text { the } \\
\text { study } \\
\text { (Study } \\
\text { ID) }\end{array}$} & \multirow{2}{*}{$\begin{array}{l}\text { Sample } \\
\text { size } \\
(\mathrm{M} / \mathrm{F})\end{array}$} & \multirow{2}{*}{$\begin{array}{l}\text { Age } \\
\text { (Mean } \pm \\
\text { SD) }\end{array}$} & \multirow[t]{2}{*}{$\begin{array}{l}\text { Type of } \\
\text { depression }\end{array}$} & \multirow[t]{2}{*}{ Acupoint } & \multirow[t]{2}{*}{ Outcome } & \multicolumn{2}{|c|}{$\begin{array}{l}\text { HAMD scores (Mean } \\
\pm \text { SD) }\end{array}$} \\
\hline & & & & & & Baseline & $\begin{array}{l}\text { Post- } \\
\text { treatment }\end{array}$ \\
\hline \multirow{2}{*}{$\begin{array}{l}\text { E: Chi } \\
\text { H } 2011 \\
{[30]}\end{array}$} & \multirow{3}{*}{$\begin{array}{l}30 \\
29\end{array}$} & \multirow{3}{*}{$\begin{array}{l}51.63 \pm \\
1.72 \\
50.36 \pm \\
7.94\end{array}$} & \multirow[t]{3}{*}{ Secondary } & \multirow{3}{*}{$\begin{array}{l}\text { DU20, DU29, } \\
\text { EX-HN1, } \\
\text { LR14, LR3, } \\
\text { KI3, ST36, } \\
\text { SP6 } \\
\text { DU20, DU29, } \\
\text { LR3, HT7, } \\
\text { PC6 }\end{array}$} & \multirow{3}{*}{$\begin{array}{l}\text { HAMD-24 } \\
\text { 4/8 weeks }\end{array}$} & $\begin{array}{l}27.26 \pm \\
4.33\end{array}$ & $\begin{array}{l}11.52 \pm \\
5.35\end{array}$ \\
\hline & & & & & & $\begin{array}{l}26 \pm \\
5.16\end{array}$ & $\begin{array}{l}15.62 \pm \\
2.86\end{array}$ \\
\hline $\begin{array}{l}\text { C: Liu } \\
\text { H } 2019 \\
{[56]}\end{array}$ & & & & & & & \\
\hline \multirow{2}{*}{$\begin{array}{l}\text { E: } \\
\text { Duan } \\
\text { DM } \\
2005 \\
{[22]}\end{array}$} & $6 / 14$ & \multirow{3}{*}{$\begin{array}{l}46.1 \pm \\
14.5 \\
69.2 \pm \\
6.9\end{array}$} & \multirow[t]{3}{*}{ Primary } & \multirow{3}{*}{$\begin{array}{l}\text { DU20, EX- } \\
\text { HN1, DU29, } \\
\text { PC6, LR3, } \\
\text { SP6 } \\
\text { DU20, DU24, } \\
\text { DU29, EX- } \\
\text { HN1, LR3, } \\
\text { HT7 }\end{array}$} & \multirow{3}{*}{$\begin{array}{l}\text { HAMD-24 } \\
6 \text { weeks }\end{array}$} & $30.1 \pm$ & $15.8 \pm 6.1$ \\
\hline & $10 / 10$ & & & & & $27.36 \pm$ & $\begin{array}{l}19.56 \pm \\
5.91\end{array}$ \\
\hline $\begin{array}{l}\text { C: } \\
\text { Cheng } \\
\text { Y } 2006 \\
{[46]}\end{array}$ & & & & & & & \\
\hline \multirow{3}{*}{$\begin{array}{l}\text { E: Gao } \\
\text { C } 2011 \\
{[23]} \\
\text { C: Ren } \\
\text { SC } \\
2019 \\
{[57]}\end{array}$} & $12 / 18$ & \multirow{3}{*}{$\begin{array}{l}45.23 \pm \\
9.93\end{array}$} & \multirow[t]{3}{*}{ Primary } & \multirow{2}{*}{$\begin{array}{l}\text { GV20, EX- } \\
\text { HN1, EX-HN3, } \\
\text { HT7, PC6, } \\
\text { SP6, ST36 }\end{array}$} & \multirow{3}{*}{$\begin{array}{l}\text { HAMD-24 } \\
6 \text { weeks }\end{array}$} & $22.23 \pm$ & $\begin{array}{l}13.43 \pm \\
521\end{array}$ \\
\hline & $5 / 25$ & & & & & $23.1 \pm$ & $19.7 \pm$ \\
\hline & & & & $\begin{array}{l}\text { HT7, PC7, } \\
\text { PC6, LR14, } \\
\text { BL15, LI4, } \\
\text { LR3, LR2, } \\
\text { BL18 }\end{array}$ & & & \\
\hline \multirow{2}{*}{$\begin{array}{l}\text { E: Yu } \\
\text { YS } \\
2012 \\
{[25]}\end{array}$} & $11 / 21$ & \multirow{3}{*}{$\begin{array}{l}36.5 \pm \\
11.3 \\
40.53 \pm \\
3.38\end{array}$} & \multirow[t]{3}{*}{ Primary } & \multirow{2}{*}{$\begin{array}{l}\text { DU20, DU29, } \\
\text { HT7, PC6, } \\
\text { BL18, GB20, } \\
\text { LI4, LR3 }\end{array}$} & \multirow{2}{*}{$\begin{array}{l}\text { HAMD-24 } \\
6 \text { weeks }\end{array}$} & $32.15 \pm$ & $9.48 \pm 4.7$ \\
\hline & 28 & & & & & $30.12 \pm$ & $\begin{array}{l}19.12 \pm \\
7.83\end{array}$ \\
\hline $\begin{array}{l}\text { C: Cai } \\
\text { MY } \\
2014 \\
{[67]}\end{array}$ & & & & $\begin{array}{l}\text { LR3, GB37, } \\
\text { PC7, SJ5 }\end{array}$ & & & \\
\hline
\end{tabular}

HAMD: Hamilton Depression Scale; M: Male; F: Female; T: Treatment; C: Control; SD: Standard Deviation; Cl: confidence level. 


\begin{tabular}{|c|c|c|c|c|c|c|c|}
\hline \multirow{2}{*}{$\begin{array}{l}\text { Pair of } \\
\text { the } \\
\text { study } \\
\text { (Study } \\
\text { ID) }\end{array}$} & \multirow{2}{*}{$\begin{array}{l}\text { Sample } \\
\text { size } \\
\text { (M/F) }\end{array}$} & \multirow{2}{*}{$\begin{array}{l}\text { Age } \\
\text { (Mean } \pm \\
\text { SD) }\end{array}$} & \multirow[t]{2}{*}{$\begin{array}{l}\text { Type of } \\
\text { depression }\end{array}$} & \multirow[t]{2}{*}{ Acupoint } & \multirow[t]{2}{*}{ Outcome } & \multicolumn{2}{|c|}{$\begin{array}{l}\text { HAMD scores (Mean } \\
\pm \text { SD) }\end{array}$} \\
\hline & & & & & & Baseline & $\begin{array}{l}\text { Post- } \\
\text { treatment }\end{array}$ \\
\hline \multirow{2}{*}{$\begin{array}{l}\text { E: Su } \\
\text { PY } \\
2013 \\
{[27]}\end{array}$} & $17 / 13$ & $58 \pm 8$ & \multirow[t]{3}{*}{ Secondary } & \multirow{2}{*}{$\begin{array}{l}\text { DU26, DU20, } \\
\text { DU24, DU16, } \\
\text { DU14, DU11 }\end{array}$} & HAMD-24 & \multirow{2}{*}{$\begin{array}{l}29.43 \pm \\
9.83\end{array}$} & \multirow{2}{*}{$\begin{array}{l}18.93 \pm \\
6.49\end{array}$} \\
\hline & \multirow[t]{2}{*}{$14 / 16$} & \multirow{2}{*}{$\begin{array}{l}63.16 \pm \\
6.21\end{array}$} & & & \multirow{2}{*}{$\begin{array}{l}4 \\
\text { weeks/30 } \\
\text { days }\end{array}$} & & \\
\hline $\begin{array}{l}\text { C: Wu } \\
\text { JX } \\
2010 \\
{[63]}\end{array}$ & & & & $\begin{array}{l}\text { BG13, DU24, } \\
\text { PC6, SP4 }\end{array}$ & & $\begin{array}{l}32.06 \pm \\
4.93\end{array}$ & $\begin{array}{l}24.86 \pm \\
3.92\end{array}$ \\
\hline
\end{tabular}

\section{Re-analysis from the unpaired-study}

The results of pooling data from both experimental and control acupuncture groups from 61 studies showed there was significant statistical difference of changed HAMD-17/24 scores after four weeks and six weeks treatment $[7-31,33-55,57-69]$. Details of the characteristics of the unpaired-studies were listed in Table 2. 
Table 2

The characteristics of the unpaired included 37 studies

\begin{tabular}{|c|c|c|c|c|c|c|}
\hline $\begin{array}{l}\text { Study } \\
\text { ID }\end{array}$ & $\begin{array}{l}\text { Sample } \\
\text { size } \\
\text { (M/F) }\end{array}$ & $\begin{array}{l}\text { Age } \\
\text { (Mean } \\
\pm \text { SD) }\end{array}$ & $\begin{array}{l}\text { Type of } \\
\text { depression }\end{array}$ & Acupoint & $\begin{array}{l}\text { HAMD } \\
\text { Scales }\end{array}$ & $\begin{array}{l}\text { Treatment } \\
\text { duration } \\
\text { (weeks) }\end{array}$ \\
\hline
\end{tabular}

2.1 Acupuncture as intervention

\begin{tabular}{lllllll} 
Han P & 27 & - & Primary & GB20, LR3 & 17 & 4 \\
$2016[7]$ & & & & & \\
$\begin{array}{l}\text { Zhang } \\
\text { J2013 } \\
{[8]}\end{array}$ & 60 & - & Primary & $\begin{array}{l}\text { BL13, BL15, BL18, BL20, BL23, } \\
\text { BL17 }\end{array}$ & 17 & 4 \\
\hline
\end{tabular}

\begin{tabular}{|c|c|c|c|c|c|}
\hline $\begin{array}{l}\text { Lv HB } \\
2018 \\
{[10]}\end{array}$ & $12 / 18$ & $\begin{array}{l}39.23 \\
\pm 9.30\end{array}$ & Primary & GV20, GV24, EX-HN3 & 17 \\
\hline
\end{tabular}

$\begin{array}{lllllrr}\text { Xu FM } & 6 / 15 & 34.00 & \text { Primary } & \text { DU20, PC6 } & 17 & 6 \\ 2009 & & & & \end{array}$

[11]

$\begin{array}{lllllll}\text { Xu W } & 10 / 15 & 45.16 & \text { Primary } & \text { DU20, EX-HN1, EX-HN5, DU26, } & 17 & 4,6 \\ 2010 & & \pm 6.96 & & \text { PC6, HT7 } & & \\ {[12]} & & & & \end{array}$

$\begin{array}{lllllll}\text { Fu WB } & 37 / 69 & 41.41 & \text { Primary } & \text { PC6, SJ5, DU20, DU29 } & 24 & 4 \\ 2008 & & \pm & & & \\ {[16]} & & 12.77 & & & \end{array}$

$\begin{array}{lllllll}\text { Liu KX } & 7 / 23 & 42.78 & \text { Primary } & \text { ST8, EX-HN5, DU20, GB20, EX- } & 24 & 4 \\ 2011 & & \pm & & \text { HN1, DU26, PC6, HT7, RN18, } & & \\ {[17]} & & 10.39 & & \text { BL18, LR3 } & \end{array}$

$\begin{array}{lllllll}\text { Wang } & 17 / 18 & 45.80 & \text { Primary } & \text { HT7, DU20, DU29, LI4, LR3 } & 24 & 4 \\ \text { QS } & & \pm 6.80 & & & & \end{array}$

2015

[19]

$\begin{array}{lllllll}\text { Xu WT } & 12 / 16 & 38.45 & \text { Primary } & \begin{array}{l}\text { SP6, GB34, PC6, DU20, KI3, } \\ \text { with electronic stimulation }\end{array} & 24 & 6 \\ 2017 & & \pm 12.7 & & \text { a } & \end{array}$

$\begin{array}{lllllll}\text { Li SW } & 11 / 19 & 40.33 & \text { Primary } & \begin{array}{l}\text { SP6, LR3, with electronic } \\ \text { stimulation }\end{array} & 24 & 4,6 \\ 2011 & & \pm & & & \end{array}$

\begin{tabular}{|c|c|c|c|c|c|}
\hline $\begin{array}{l}\text { Zhou L } \\
2011 \\
\text { [26] }\end{array}$ & $15 / 20$ & $\begin{array}{l}40.00 \\
\pm 4.00\end{array}$ & Primary & $\begin{array}{l}\text { GB20, Sixth transverse } \\
\text { foramen, Emotional area, with } \\
\text { electronic stimulation }\end{array}$ & 24 \\
\hline
\end{tabular}
Deviation; Cl: confidence level. 


\begin{tabular}{|c|c|c|c|c|c|c|}
\hline $\begin{array}{l}\text { Study } \\
\text { ID }\end{array}$ & $\begin{array}{l}\text { Sample } \\
\text { size } \\
(M / F)\end{array}$ & $\begin{array}{l}\text { Age } \\
\text { (Mean } \\
\pm \text { SD) }\end{array}$ & $\begin{array}{l}\text { Type of } \\
\text { depression }\end{array}$ & Acupoint & $\begin{array}{l}\text { HAMD } \\
\text { Scales }\end{array}$ & $\begin{array}{l}\text { Treatment } \\
\text { duration } \\
\text { (weeks) }\end{array}$ \\
\hline $\begin{array}{l}\text { Deng } \\
\text { AJ } \\
2008 \\
{[28]}\end{array}$ & 29 & $\begin{array}{l}50.03 \\
\pm 4.43\end{array}$ & Secondary & RN12, RN10, RN6, RN4 & 17 & 4 \\
\hline $\begin{array}{l}\text { Li ZF } \\
2015 \\
{[29]}\end{array}$ & 30 & $\begin{array}{l}49.80 \\
\pm 3.39\end{array}$ & Secondary & $\begin{array}{l}\text { RN4, EX-CA1, ST25, SP6, LI4, } \\
\text { LR3, DU20, DU29! }\end{array}$ & 17 & 4 \\
\hline $\begin{array}{l}\text { Yu SJ } \\
2015 \\
{[32]}\end{array}$ & 30 & $\begin{array}{l}28.00 \\
\pm \\
16.00\end{array}$ & Secondary & DU26, DU23, DU16, RN24, PC8 & 17 & 4 \\
\hline $\begin{array}{l}\text { Chen } \\
\mathrm{HL} \\
2007 \\
{[33]}\end{array}$ & 22 & $\begin{array}{l}26.70 \\
\pm 4.50\end{array}$ & Secondary & $\begin{array}{l}\text { DU20, BL15, BL18, LR14, BL20, } \\
\text { LR13 }\end{array}$ & 24 & 4 \\
\hline $\begin{array}{l}\text { Li QJ } \\
2019 \\
{[34]}\end{array}$ & $9 / 25$ & $\begin{array}{l}44.38 \\
\pm \\
12.79\end{array}$ & Primary & $\begin{array}{l}\text { DU20, DU29, RN17, HT7, PC7, } \\
\text { PC6, SP6, ST36, BL15, BL20, } \\
\text { LI4, LR3, BL62 }\end{array}$ & 24 & 4 \\
\hline $\begin{array}{l}\text { Liu Q } \\
2019 \\
{[35]}\end{array}$ & $4 / 26$ & $\begin{array}{l}53.16 \\
\pm 8.32\end{array}$ & Primary & $\begin{array}{l}\text { DU29, ST36, RN13, RN12, } \\
\text { RN11, RN6, ST25, PC6, DU20, } \\
\text { DU24 }\end{array}$ & 17 & 6 \\
\hline \multicolumn{7}{|c|}{ 2.2 Acupuncture as control } \\
\hline $\begin{array}{l}\text { Li JC } \\
2011 \\
{[39]}\end{array}$ & $14 / 6$ & $\begin{array}{l}42.70 \mathbb{8} \\
13.24\end{array}$ & Primary & EX-HN1, PC6, SP6 & 17 & 6 \\
\hline $\begin{array}{l}\mathrm{Li} \mathrm{C} \\
2011 \\
{[40]}\end{array}$ & $15 / 15$ & $\begin{array}{l}32.27 \\
\pm \\
10.79\end{array}$ & Primary & DU20, DU26, PC6, HT7, LR3 & 24 & 4 \\
\hline $\begin{array}{l}\mathrm{LiC} \\
2017 \\
{[42]}\end{array}$ & $13 / 15$ & $\begin{array}{l}18.00- \\
55.00\end{array}$ & Primary & DU26, PC6, HT7, LR3 & 17 & 6 \\
\hline $\begin{array}{l}\text { Wang } \\
\text { CJ } \\
2017 \\
{[43]}\end{array}$ & $7 / 23$ & $\begin{array}{l}42.17 \\
\pm \\
11.35\end{array}$ & Primary & GV20, EX-HN3, PC6, HT7, SP6 & 24 & 4 \\
\hline $\begin{array}{l}\text { Cheng } \\
\text { YZ } \\
2012 \\
{[44]}\end{array}$ & $15 / 15$ & $\begin{array}{l}34.73 \\
\pm 8.37\end{array}$ & Primary & DU26, HT7, PC6, LR3 & 24 & 6 \\
\hline
\end{tabular}

HAMD: Hamilton Depression Scale; M: Male; F: Female; T: Treatment; C: Control; SD: Standard Deviation; Cl: confidence level. 


\begin{tabular}{|c|c|c|c|c|c|c|}
\hline $\begin{array}{l}\text { Study } \\
\text { ID }\end{array}$ & $\begin{array}{l}\text { Sample } \\
\text { size } \\
(M / F)\end{array}$ & $\begin{array}{l}\text { Age } \\
\text { (Mean } \\
\pm \text { SD) }\end{array}$ & $\begin{array}{l}\text { Type of } \\
\text { depression }\end{array}$ & Acupoint & $\begin{array}{l}\text { HAMD } \\
\text { Scales }\end{array}$ & $\begin{array}{l}\text { Treatment } \\
\text { duration } \\
\text { (weeks) }\end{array}$ \\
\hline $\begin{array}{l}\text { Du YH } \\
2005 \\
{[47]}\end{array}$ & $10 / 30$ & $\begin{array}{l}42.80 \\
\pm \\
14.90\end{array}$ & Primary & $\begin{array}{l}\text { LR14, LR3, GB34, SJ6, PC6, } \\
\text { ST36 }\end{array}$ & 24 & 6 \\
\hline $\begin{array}{l}\text { Fan L } \\
2009 \\
{[49]}\end{array}$ & $16 / 13$ & $\begin{array}{l}34.73 \\
\pm 8.37\end{array}$ & Primary & DU26, HT7, PC6, LR3 & 24 & 4 \\
\hline $\begin{array}{l}\text { Su H } \\
2010 \\
{[50]}\end{array}$ & $5 / 23$ & $\begin{array}{l}41.10 \\
\pm \\
11.50\end{array}$ & Primary & LR3, SP6, PC6, HT7 & 24 & 4 \\
\hline $\begin{array}{l}\text { Yu XP } \\
2016 \\
{[51]}\end{array}$ & $14 / 16$ & $\begin{array}{l}53.00 \\
\pm 7.00\end{array}$ & Secondary & $\begin{array}{l}\text { DU29, DU20, EX-HN1, GB20, } \\
\text { EX-HN5, PC6, HT7, PC6 }\end{array}$ & 24 & 4 \\
\hline $\begin{array}{l}\text { Min XR } \\
2014 \\
\text { [52] }\end{array}$ & $26 / 14$ & $\begin{array}{l}42.00- \\
69.00\end{array}$ & Secondary & $\begin{array}{l}\text { DU20, PC6, SP6, DU29, HT7, } \\
\text { RN17, KI3 }\end{array}$ & 24 & 4 \\
\hline $\begin{array}{l}\text { Yu XP } \\
2016 \mathrm{~b} \\
{[53]}\end{array}$ & $10 / 15$ & $\begin{array}{l}56.64 \\
\pm 7.59\end{array}$ & Secondary & $\begin{array}{l}\text { GB20, LI11, SJ5, LI4, GB30, } \\
\text { GB34, ST36, ST41, BL60, RN17 }\end{array}$ & 17 & 4 \\
\hline $\begin{array}{l}\text { Feng } \\
\text { LM } \\
2012 \\
{[54]}\end{array}$ & $5 / 15$ & $\begin{array}{l}54.05 \\
\pm 6.40\end{array}$ & Secondary & $\begin{array}{l}\text { LR14, LR3, GB34, SJ6, PC6, } \\
\text { ST36 }\end{array}$ & 24 & 4 \\
\hline $\begin{array}{l}\text { Li ZH } \\
2019 \\
{[55]}\end{array}$ & $12 / 24$ & $\begin{array}{l}38.28 \\
\pm 9.65\end{array}$ & Primary & $\begin{array}{l}\text { DU20, DU29, SP6, PC6, HT7, } \\
\text { LR3 }\end{array}$ & 24 & 6 \\
\hline $\begin{array}{l}\text { Wang } \\
\text { HY } \\
2019 \\
{[58]}\end{array}$ & $16 / 14$ & $\begin{array}{l}51.53 \\
\pm 8.08\end{array}$ & Primary & $\begin{array}{l}\text { DU26, DU20, PC6, HT7, RN17, } \\
\text { LR3, LR2, GB43 }\end{array}$ & 24 & 4 \\
\hline $\begin{array}{l}\text { Xu P } \\
2019 \\
{[59]}\end{array}$ & $14 / 21$ & $\begin{array}{l}43.57 \\
\pm \\
10.86\end{array}$ & Primary & $\begin{array}{l}\text { LR14, LR3, ST40, BL20, ST36, } \\
\text { RN22 }\end{array}$ & 24 & 6 \\
\hline $\begin{array}{l}\text { Zhang } \\
\text { LX } \\
2019 \\
{[60]}\end{array}$ & $11 / 16$ & $\begin{array}{l}72.22 \\
\pm 5.83\end{array}$ & Primary & DU200DU29 & 17 & 4 \\
\hline $\begin{array}{l}\text { Wu J } \\
2016 \\
{[61]}\end{array}$ & $8 / 28$ & $\begin{array}{l}61.62 \\
\pm 6.52\end{array}$ & Secondary & PC6, DU26, SP6 & 17 & 4 \\
\hline
\end{tabular}

HAMD: Hamilton Depression Scale; M: Male; F: Female; T: Treatment; C: Control; SD: Standard Deviation; Cl: confidence level. 


\begin{tabular}{|c|c|c|c|c|c|c|}
\hline $\begin{array}{l}\text { Study } \\
\text { ID }\end{array}$ & $\begin{array}{l}\text { Sample } \\
\text { size } \\
(\mathrm{M} / \mathrm{F})\end{array}$ & $\begin{array}{l}\text { Age } \\
\text { (Mean } \\
\pm \text { SD) }\end{array}$ & $\begin{array}{l}\text { Type of } \\
\text { depression }\end{array}$ & Acupoint & $\begin{array}{l}\text { HAMD } \\
\text { Scales }\end{array}$ & $\begin{array}{l}\text { Treatment } \\
\text { duration } \\
\text { (weeks) }\end{array}$ \\
\hline $\begin{array}{l}\text { Zhang } \\
\text { ZR } \\
2013 \\
{[62]}\end{array}$ & $17 / 13$ & $\begin{array}{l}61.43 \\
\pm 9.91\end{array}$ & Secondary & DU20, DU18, BG13, GB9 & 24 & 4,6 \\
\hline $\begin{array}{l}\text { Zhang } \\
\text { XL } \\
2019 \\
{[64]}\end{array}$ & $7 / 23$ & $\begin{array}{l}43.77 \\
\pm \\
11.14\end{array}$ & Primary & $\begin{array}{l}\text { DU20, DU29, HT7, LR3, PC6, } \\
\text { RN17, BL15, BL20 }\end{array}$ & 17 & 4 \\
\hline $\begin{array}{l}\text { Su PZ } \\
2011 \\
\text { [68] }\end{array}$ & 20 & $\begin{array}{l}20.30 \\
\pm 3.13\end{array}$ & Secondary & $\begin{array}{l}\text { DU20, DU24, PC6, LI4, ST36, } \\
\text { SP6, LR3 }\end{array}$ & 17 & 6 \\
\hline
\end{tabular}

The t test results (see Table 3 and Fig. 3) showed there was significant statistical difference between experimental and control acupuncture at four weeks of HAMD-17 $(t=2.54, P=0.01, M D=4.86)$, four weeks of HAMD-24 $(t=2.76, P=0.006, M D=3.90)$, and six weeks of HAMD-24 $(t=3.35, P=0.0009, M D=$ 6.57). No statistical difference was found only between groups in six weeks of HAMD-17 $(t=1.69, P=$ $0.09, \mathrm{MD}=3.38$ ). 
Table 3

$t$-test results of the comparison between experimental and control acupuncture groups

\begin{tabular}{|c|c|c|c|c|c|c|c|c|c|}
\hline \multirow[t]{2}{*}{ Outcome } & \multicolumn{3}{|c|}{ Experimental acupuncture } & \multicolumn{3}{|c|}{ Control acupuncture } & \multirow{2}{*}{$\begin{array}{l}\text { Effect } \\
\text { estimates } \\
(\mathrm{MD} \\
95 \% \mathrm{Cl})\end{array}$} & \multirow[t]{2}{*}{$t$} & \multirow[t]{2}{*}{$P$} \\
\hline & $\begin{array}{l}\text { Effect } \\
\text { estimates } \\
(\mathrm{MD}, \\
95 \% \mathrm{Cl})\end{array}$ & $\begin{array}{l}\text { Sample } \\
\text { size }\end{array}$ & $1^{2}$ & $\begin{array}{l}\text { Effect } \\
\text { estimates } \\
(\mathrm{MD}, \\
95 \% \mathrm{Cl})\end{array}$ & $\begin{array}{l}\text { Sample } \\
\text { size }\end{array}$ & $1^{2}$ & & & \\
\hline $\begin{array}{l}\text { HAMD- } \\
17 \text { in } 4 \\
\text { weeks }\end{array}$ & $\begin{array}{l}11.41 \\
{[8.30,} \\
14.51]\end{array}$ & 283 & $96 \%$ & $\begin{array}{l}6.55 \\
{[4.66} \\
8.44]\end{array}$ & 246 & $91 \%$ & $\begin{array}{l}4.86 \\
{[1.24,8.48]}\end{array}$ & 2.54 & 0.01 \\
\hline $\begin{array}{l}\text { HAMD- } \\
17 \text { in } 6 \\
\text { weeks }\end{array}$ & $\begin{array}{l}12.23 \\
{[9.50,} \\
14.97]\end{array}$ & 188 & $91 \%$ & $\begin{array}{l}8.85 \\
{[6.08,} \\
11.61]\end{array}$ & 149 & $95 \%$ & $\begin{array}{l}3.38 \\
{[-0.48,} \\
7.24]\end{array}$ & 1.69 & $0.0 \varsigma$ \\
\hline $\begin{array}{l}\text { HAMD- } \\
24 \text { in } 4 \\
\text { weeks }\end{array}$ & $\begin{array}{l}12.30 \\
{[10.05,} \\
14.54]\end{array}$ & 465 & $95 \%$ & $\begin{array}{l}8.40 \\
{[7.05} \\
9.75]\end{array}$ & 377 & $81 \%$ & $\begin{array}{l}3.90[1.28, \\
6.52]\end{array}$ & 2.76 & $\begin{array}{l}< \\
0.01\end{array}$ \\
\hline $\begin{array}{l}\text { HAMD- } \\
24 \text { in } 6 \\
\text { weeks }\end{array}$ & $\begin{array}{l}16.31 \\
\text { [11.30, } \\
21.31]\end{array}$ & 166 & $96 \%$ & $\begin{array}{l}8.87 \\
{[7.13} \\
10.62]\end{array}$ & 305 & $88 \%$ & $\begin{array}{l}6.57[1.43, \\
11.71]\end{array}$ & 3.35 & $\begin{array}{l}< \\
0.01\end{array}$ \\
\hline
\end{tabular}

\section{Discussion}

According to our study, the effectiveness of acupuncture for decreasing the severity of depression was superior in trials which considered acupuncture as experimental treatment compared to those of the acupuncture as control. The difference of post-treatment HAMD-17 scales and HAMD-24 scales were average -2.95 cores and -5.55 cores, which were both statistical and clinical meaningful for relieving depression.

This study attempts to quantify the impact of performance and detection bias in acupuncture clinical trials. The application of paired meta-analysis can reduce the system error of indirect comparison as much as possible, and re-analysis from the unpaired-study further confirmed the stability of the results. The data of this study can explain to some extent the consequences of destroying the uncertainty principle caused by the lack of blinding method in the non-pharmaceutical therapy researches represented by acupuncture.

However, since we used indirect comparisons, the strength of the evidence was weakened. Although we used pairing method to reduce the influence of confounding factors, the results of meta-analysis had a certain degree of statistical heterogeneity due to the inconsistency of acupoint selection between acupuncture studies. 
As the result showed in the study, we suggest that consideration should be given to existing evidence of acupuncture for depression. When acupuncture is used in the experimental group, the effect of acupuncture may be overestimated; but it may be under estimated when acupuncture appearing in the control group. It is suggested that the actual effectiveness of acupuncture should be carefully considered when referring to the corresponding evidence in clinical practice. Similar consequences may appear in other diseases, as doctors' personal preference could lead to performance and detection bias, therefore implicates study results. Moreover, it can be speculated that detection bias could cause due to patients' self-assessment, which is highly subjective.

According to this study, blinding method, applied with extra means to ensure its success, is the key solution to avoid performance and detection bias during acupuncture clinical trials. We suggest that future trials should either use adequate random allocation and concealment, blinding the outcome assessors and statisticians, or improve the monitoring mechanism, so that the research designer does not intervene in the implementation process of intervention and does not directly contact patients, so as to reduce the impact of bias on the research. However, blinding method is hard to be used in the nonplacebo-controlled trial. Thus, some special types of randomized trials can be used in acupuncture clinical trial. For example, sequential multiple assignment randomized trial can improve external authenticity and get closer to the real treatment process through building and comparing dynamic treatment regimens [7072]. In previous studies, we also proposed the feasibility and advantages of partially randomized patient preference trial adopted in evaluation of non-drug therapy (e.g. Acupuncture) by analyzing the application status of modified clinical trials considered patients' preferences [73, 74].

\section{Conclusions}

This study found that the effectiveness of acupuncture for decreasing the severity of depression was superior in trials those employed acupuncture as experimental treatment, which showed that performance and detection bias caused by the absence of blinding method played an important role in acupuncture clinical trials.

\section{Abbreviations}

$\mathrm{Cl}$

confidence interval

HAMD

Hamilton Depression Scale

MD

Mean Difference

SD

standard deviation

\section{Declarations}




\section{Ethics approval and consent to participate}

Not applicable.

Consent for publication

Not applicable.

\section{Availability of data and materials}

The authors confirm that the data supporting the findings of this study are available within the article and its supplementary information files.

\section{Competing interests}

The authors declare that they have no competing interests.

\section{Funding}

Hui-Juan Cao is supported by the National Natural Science Foundation of China (81804000). The funders had no role in study design, data collection and analysis, decision to publish, or preparation of the manuscript.

\section{Authors' contributions}

RTW and LJC analyzed basic characteristics of trials and analyzed data, and were major contributors in writing the manuscript. JPL and YTF assisted with the research methods and result explanation. MH participated in research designing, data analyzing and interpretation of indicators. HLC ensure that original data, original figures, materials and code upon which the submission is based are preserved following best practices in the field so that they are retrievable for reanalysis; and confirm that data, figures, materials and code presentation accurately reflects the original; and foresee and minimize obstacles to the sharing of data, materials, code described in the work. HLC also ensure that the entire author group is fully aware of and in compliance with best practices in the discipline of publication. All authors read and approved the final manuscript.

\section{Acknowledgements}

Not applicable.

\section{References}


1. Benjamin Djulbegovic CL, Bennett, Jared R, Adams GH, Lyman. The uncertainty principle and industrysponsored research. Lancet. 2000;356(9230):635-8.

2. Barcot, Ognjen, et al. Risk of bias assessments for blinding of participants and personnel in Cochrane reviews were frequently inadequate. J Clin Epidemiol. 2019;113:104-13.

3. Liu Jianping KL, Lotte G, Christian. Misuse of randomization: a review of Chinese randomized trials of herbal medicines for chronic hepatitis B. The American Journal of Chinese Medicine. 2002;30(1):173-6.

4. Lingling $Y$, et al. A systematic review of acupuncture and Chinese herbal medicine for postpartum depression. Complementary Therapies in Clinical Practice. 2018;33:85-92.

5. Li Q, Xia Y, Wang YY, Liu JP. Reevaluation on quality of TCM systematic reviews and meta-analyses documents published in domestic Chinese journals. Journal of Beijing University of traditional Chinese medicine (clinical version of traditional Chinese Medicine). 2012, 19 (3): 28-33.

6. Armour M, et al. Acupuncture for depression: A systematic review and meta-analysis. J Clin Med. 2019;8(8):1140.

7. Han P, Wang ZY, Huang YS, Luo QL. Clinical observation of post-stroke depression by acupuncture and study P300 with events related potential. Journal of Clinical Medical. 2016;3(44):8753-5.

8. Zhang J, et al. A multicenter randomized controlled study of acupuncture for mild to moderate depression. Compilation of Papers of the 2013 Annual Conference of the Department of Psychiatry. 2013: 163-168.

9. Liu P. The clinical observation of acupuncture combine with baidu and neiguan on depression. Dissertation for Master Degree of Heilongjiang University of Chinese Medicine. 2009.

10. Lv HB, Gan SY. Clinical Observation on treatment of 30 cases of depression with head point acupuncture. Jiangsu Journal of Traditional Chinese Medicine. 2018;50(01):56-8.

11. Xu FM, Wang Q, Liu XL. Clinical observation on acupuncture treatment of depression. Journal of Clinical Acupuncture Moxibustion. 2009;25(09):27-8.

12. Xu W. The clinical study of "Tiao Shen Jie Yu" acupuncture on depression of stagnatio of the liver-qi. Dissertation for Master Degree of Heilongjiang University of Chinese Medicine. 2010.

13. Zhou X. Acupuncture treatment of 32 cases of depression. Hunan Journal of traditional Chinese medicine. 2011;27(05):53-4.

14. Wang XF, Zhang XP, Zhao ZG. Electroacupuncture for depression and its effect on plasma adrenocorticotropic hormone and cortisol. Journal of Sichuan of Traditional Chinese Medicine. 2007 (04): 102-103.

15. Chen CW. Clinical observation on treating neurotic depression between fluoxetine and acupuncture. Dissertation for Master Degree of Guangzhou University of Chinese Medicine. 2007.

16. Fu WB. A randomized controlled study on acupuncture treatment of regulating liver and spirit for depression and the post-receptor signal transduction mechanism. Dissertation for Doctor Degree of Hunan University of Chinese Medicine. 2008. 
17. Liu KX. The clinical observation of acupuncture treatment in depression. Dissertation for Master Degree of Heilongjiang University of Chinese Medicine. 2011.

18. Wang C. Study of clinical effect and improvement of TCM syndromes of acupuncture for depression with liver-qi stagnation syndrome. Dissertation for Master Degree of Guangdong University of Chinese Medicine. 2007.

19. Wang QS, Ji XD, Yuan GZ, Zhu WX. Clinical observation of acupuncture treatment of depression with insomnia and analysis of its effect on plasma 5-HT content. Journal of Clinical Acupuncture Moxibustion. 2015;31(11):8-10.

20. Wang QS, Ji XD, Zhu WX, Yu HY. Effect of acupuncture on the serum brain-derived neurotrophic factor level in depressive patients with hyperactivity of 'Fire' due to 'Yin' deficiency. Journal of Capital Medical University. 2016;37(02):176-80.

21. Xu WT. The clinical effectiveness of electro-acupuncture on depression and study on its action mechanism. Dissertation for Doctor Degree of Guangdong University of Chinese Medicine. 2017.

22. Duan DM. Study on clinical and mechanism of electroacupuncture anti-depression. Dissertation for Master Degree of Beijing University of Chinese Medicine. 2005.

23. Gao C. Clinical study on acupuncture treatment of mild to moderate depression. Dissertation for Master Degree of Liaoning University of Chinese Medicine. 2011.

24. Li SW. Clinical research on depression of liver-depression and spleen-deficiency with electroacupuncture treatment of fixed-frequency. Dissertation for Master Degree of Guangzhou University of Chinese Medicine. 2011.

25. Yu YS. The clinical study of using the acupuncture reduction manipulation method in treating depression of the liver qi stagnation syndrome. Dissertation for Master Degree of Guangzhou University of Chinese Medicine. 2012.

26. Zhou L. Clinical study of the electro-nape acupuncture in the treatment of depression. Dissertation for Master Degree of Heilongjiang University of Chinese Medicine. 2011.

27. Su PY, et al. Post-stroke depression treated with acupuncture therapy of dredging governor vessel and regulating mentality: a randomized controlled trial. Chinese Acupuncture Moxibustion. 2013;33(01):3-7.

28. Deng AJ. A clinic study on the treatment of involutional depression with ventro-acupuncture of bo-shi. Dissertation for Master Degree of Guangzhou University of Chinese Medicine. 2008.

29. Li ZF. The clinical research of electroacupuncture on mild to moderate perimenopausal depression. Dissertation for Doctor Degree of Guangzhou University of Chinese Medicine. 2015.

30. Chi H, Zou W. Yishen tiaoan acupuncture therapy in the treatment of perimenopausal depression: An Observation of 30 Cases. Journal of Clinical Acupuncture Moxibustion. 2011;27(07):4-7.

31. Li HB. Clinical observation on the treatment of kidney deficiency and liver stagnation of perimenopausal depression by acupuncture. Dissertation for Master Degree of Heilongjiang University of Chinese Medicine. 2015. 
32. Yu SJ, Li XQ, Feng XM, Cao WF. Therapeutic efficacy of acupuncture at the thirteen ghost points for postpartum depression and its effect on the quality of life. Shanghai Journal of Acupuncture Moxibustion. 2015;34(01):14-6.

33. Chen HL. The back-shu and the front-mu combining source and network points suppository for the treatment of the primary dysmenorrhea. Dissertation for Master Degree of Heilongjiang University of Chinese Medicine. 2007.

34. Li QJ. Clinical therapeutic effect of ping wan li zhi acupuncture manipulation in the treatment of depression due to the deficiency of heart and spleen. Dissertation for Master Degree of Yunnan University of Chinese Medicine. 2019.

35. Liu Q. Clinical Observation on the treatment of chronic insomnia with depression by yintang periosteal acupuncture combined with "Old Ten Needles". Dissertation for Master Degree of Inner Mongolia Medical University. 2019.

36. Xie M. The clinical research on effect of spirit-reinforcing and qi-regulating acupuncture therapy on subthreshold depression patients with qi-stagnation. Dissertation for Master Degree of Beijing University of Chinese Medicine. 2018.

37. Chen YY. Clinical research of Bo's abdominal combined with body acupuncture treated mild and midrange depression. Dissertation for Doctor Degree of Southern Medical University. 2011.

38. Cheng XF. The observation of acupuncture neiguan sanyinjiao in treatment of diarrhea type irritable bowel syndrome with depressive tendencies. Dissertation for Master Degree of Shanxi University of Chinese Medicine. 2017.

39. Li JC. The clinical research on the depression with electro-"DI" acupuncture. Dissertation for Master Degree of Heilongjiang University of Chinese Medicine. 2011.

40. Li C. To observe the clinical curative effect of needling five zang-organ shu in treating depression. Dissertation for Master Degree of Shandong University of Chinese Medicine. 2011.

41. Wang XN. Clinical comparative study of big-circle needling technique and manual acupuncture in treating mild to moderate depression with liver-qi stagnation syndromes. Dissertation for Master Degree of Dalian Medical University. 2017.

42. Li C. The clinical curative effect of prismatic method on the treatment of hepatic depression was observed. Dissertation for Master Degree of Heilongjiang University of Chinese Medicine. 2017.

43. Wang CJ. Clinical observation of scalp and body electroacupuncture in treating depression with chronic pain. Dissertation for Master Degree of Heilongjiang University of Chinese Medicine. 2017.

44. Cheng YZ. Eight methods of intelligent turtle with syndrome differentiation acupuncture on depression and quality of life in the treatment of clinical research. Dissertation for Master Degree of Shanxi University of Chinese Medicine. 2012.

45. Chen L. Clinical observation combining head emotional area and abdominal area-1 of sun's abdominal-acupuncture on the treatment foe liver-qi stagnation depression. Dissertation for Master Degree of Heilongjiang University of Chinese Medicine. 2015. 
46. Cheng Y, Tang QS. Clinical random control study of acupuncture on treating stagnation of liver-qi with deficiency of the spleen syndrome of gerontic depressive disorder after stroke. Journal of Traditional Chinese Encephalopathy. 2006;2(2):129-31.

47. Du YH, li GP, Yan H, Zhang XJ, Huang LF. Clinical study on needling method for regulating mental activities and soothing liver for treatment of melancholia. Chinese Acupuncture Moxibustion. 2005;25(03):151-4.

48. Yao XY. Comparison of the efficacy of electroacupuncture and hand acupuncture in the treatment of first-onset depression. Dissertation for Master Degree of Beijing University of Chinese Medicine. 2016.

49. Fan L, Fu WB, Zhu XP, Du Q, Wen X. Clinical study on treatment of depressive neurosis with acupuncture therapy of miraculous turtle eight meridians method. Liaoning Journal of Traditional Chinese Medicine. 2009;36(08):1383-5.

50. Su H, et al. Effect of acupuncture at baihui (GV 20) and zusanli (ST 36) on the level of serum inflammatory cytokines in patients with depression. World Journal of Acupuncture-Moxibustion. 2010;30(03):195-9.

51. Yu XP, Sun GP. Therapeutic Observation of tiao du tong ren needling for post-stroke depression. Shanghai Journal of Acupuncture Moxibustion. 2016;35(03):273-5.

52. Min XR. Clinical study of eye acupuncture therapy for post-stroke depression. Dissertation for Master Degree of Liaoning University of Chinese Medicine. 2014.

53. Yu XP, Yang CG, Zhang JY. Therapeutic effect of acupuncture at danzhong point on post - stroke depression. Journal of Clinical Acupuncture Moxibustion. 2016;32(08):59-62.

54. Feng LM, Shi J. Clinical study on tiaoshen shugan acupuncture for post-stroke depression. The 12th National Symposium on Acupuncture and Meridians of the Chinese Academy of Acupuncture and Meridians. 2012: 208-211.

55. Li ZH. Clinical observation on the treatment of depressive neurosis with tiaoshendingzhi acupuncture manipulation. Dissertation for Master Degree of Heilongjiang University of Chinese Medicine. 2019.

56. Liu H. Clinical observe of back-shu point warm acupuncture on the treatment of menopausal depression. Chinese Journal of Ethnomedicine Ethnopharmacy. 2019;28(7):103-5.

57. Ren SC. The clinical research of "Yu Ten-Point Needling" in treating mild and moderate depression of yu syndrome (Liver-qi stagnation type). Dissertation for Master Degree of North China University of Science and Technology. 2019.

58. Wang HY. Clinical observation on the treatment of depression syndrome of deficiency of heart and spleen by combined routine acupuncture and warming needle moxibustion at xinshu and pishu. Dissertation for Master Degree of Heilongjiang University of Chinese Medicine. 2019.

59. Xu P. The effect of acupuncture "Peitu Kaiyu" on treating mild and moderate depression. Dissertation for Master Degree of Beijing University of Chinese Medicine. 2019.

60. Zhang LX. A Study of electro-acupuncture treatment (Anmian and Yiming) in the neck of depression among the elderly. Dissertation for Master Degree of Beijing University of Chinese Medicine. 2019. 
61. Wu J. Effect of 5-HT, S100ß levels and HAMD, NIHSS score in patients with post-stroked depression (PSD) by prozac combined acupuncture. Chinese Journal of Biochemical Pharmaceutics. 2016;36(07):55-8.

62. Zhang ZR. The clinical research on treating post stroke depression by electric-acupuncture shen wu xing. Dissertation for Master Degree of Chengdu University of Chinese Medicine. 2013.

63. Wu JX. Clinical research of clinical symptom, objective sign of combined moxibustion and zhisanzhen (MAZ) for treating post-stroke depressive disorder (PSD) patients. Dissertation for Doctor Degree of Guangzhou University of Chinese Medicine. 2010.

64. Zhang XL. Clinical observation on the treatment of depression syndrome of deficiency of heart and spleen by combined routine acupuncture and warming needle moxibustion at xinshu and pishu. Dissertation for Master Degree of Heilongjiang University of Chinese Medicine. 2019.

65. Song MQ. The clinical study of moxibustion on the therapy of depression. Dissertation for Master Degree of Shandong University of Chinese Medicine. 2006.

66. Wang YW. Clinical study on integrated acupuncture and moxibustion regimen in treating mildtomoderate depression. Journal of New Chinese Medicine. 2019;51(10):249-52.

67. Cai MY. The clinical study of using acupuncture based on combination of yuan and luo points method and cognitive therapy on depression of the liver qi stagnation syndrome. Dissertation for Doctor Degree of Nanjing University of Chinese Medicine. 2014.

68. Su PZ, Zhang GF, Liu YY, Qu WX. Acupuncture combined with cognitive behavior therapy for the treatment of depression in youngsters with Internet addiction disorder: Clinical Study. Liaoning Journal of Traditional Chinese Medicine. 2011;38(03):532-5.

69. Jin YB, Xiang HY, Zheng LF. Influence of catgut implantation at back - shu points on HAMD score and sex hormones in patients with perimenopausal depression and anxiety. Chinese Archives of Traditional Chinese Medicine. 2013;31(06):1322-4.

70. Lavori PW, Dawson R. Dynamic treatment regimes: practical design considerations. Clin Trials. 2014;1(1):9-20.

71. Lavori PW, Dawson R, Rush AJ. Flexible treatment strategies in chronic disease: clinical and research implications. Biol Psychiatry. 2000;48(6):605-14.

72. Cao HJ, L JP. Discussion on the application of SMART design in the evaluation of curative effect of non-drug therapy of traditional Chinese medicine. Beijing Journal of Traditional Chinese Medicine. 2020;39(1):78-80.

73. Li X, Zhang Y, Liu J, Cao H. Design and application of partial randomized clinical trials considering patients' preferences in effect evaluation of Chinese medicine non-drug therapy. Journal of Traditional Chinese Medicine. 2016;57(20):1750-3, 1767.

74. Cao H, Liu J, Hu H, Wang NS. Using a partially randomized patient preference study design to evaluate the therapeutic effect of acupuncture and cupping therapy for fibromyalgia: study protocol for a partially randomized controlled trial. Trials. 2014;15:280. 
Figures

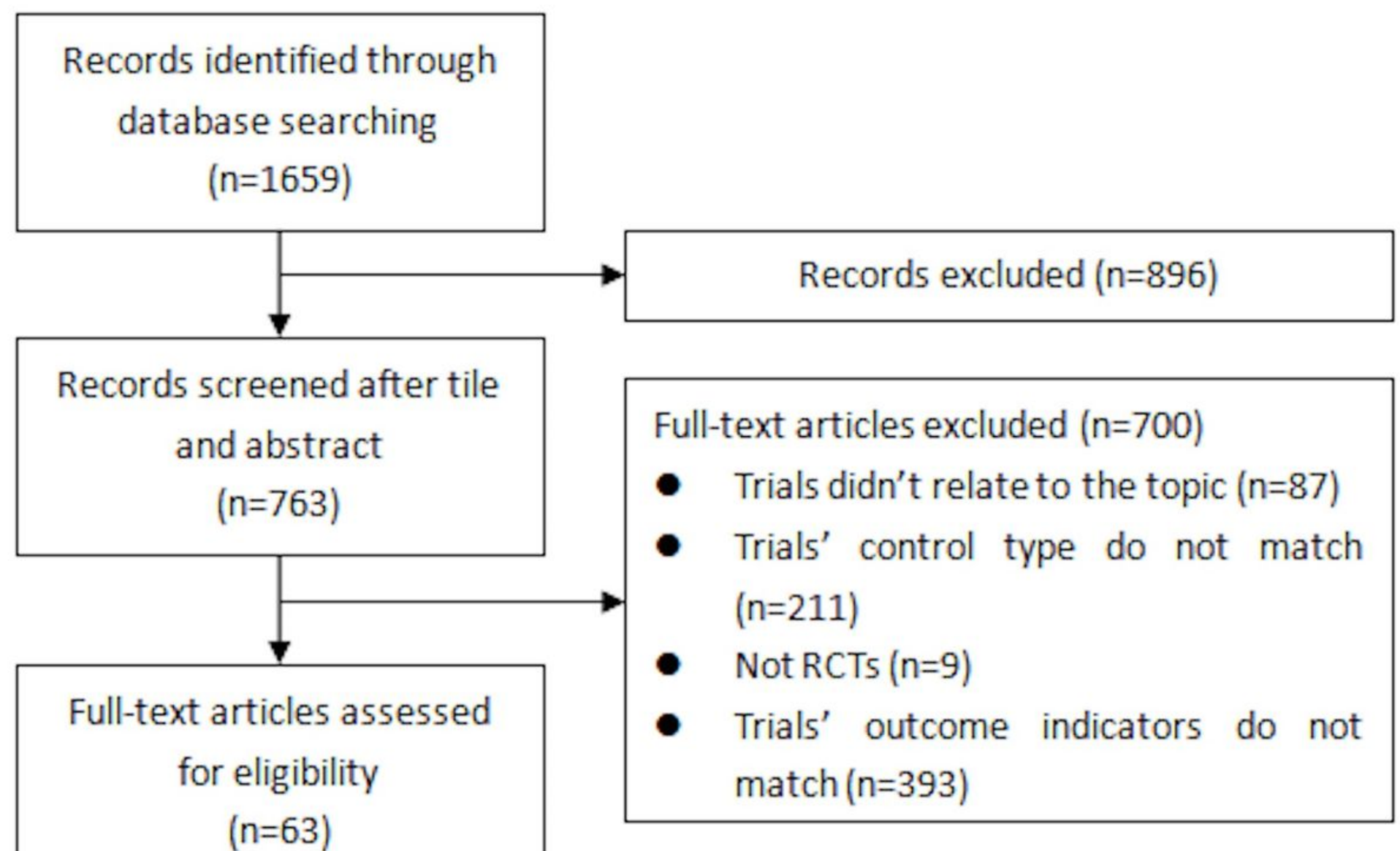

Figure 1

Study flow chart. 


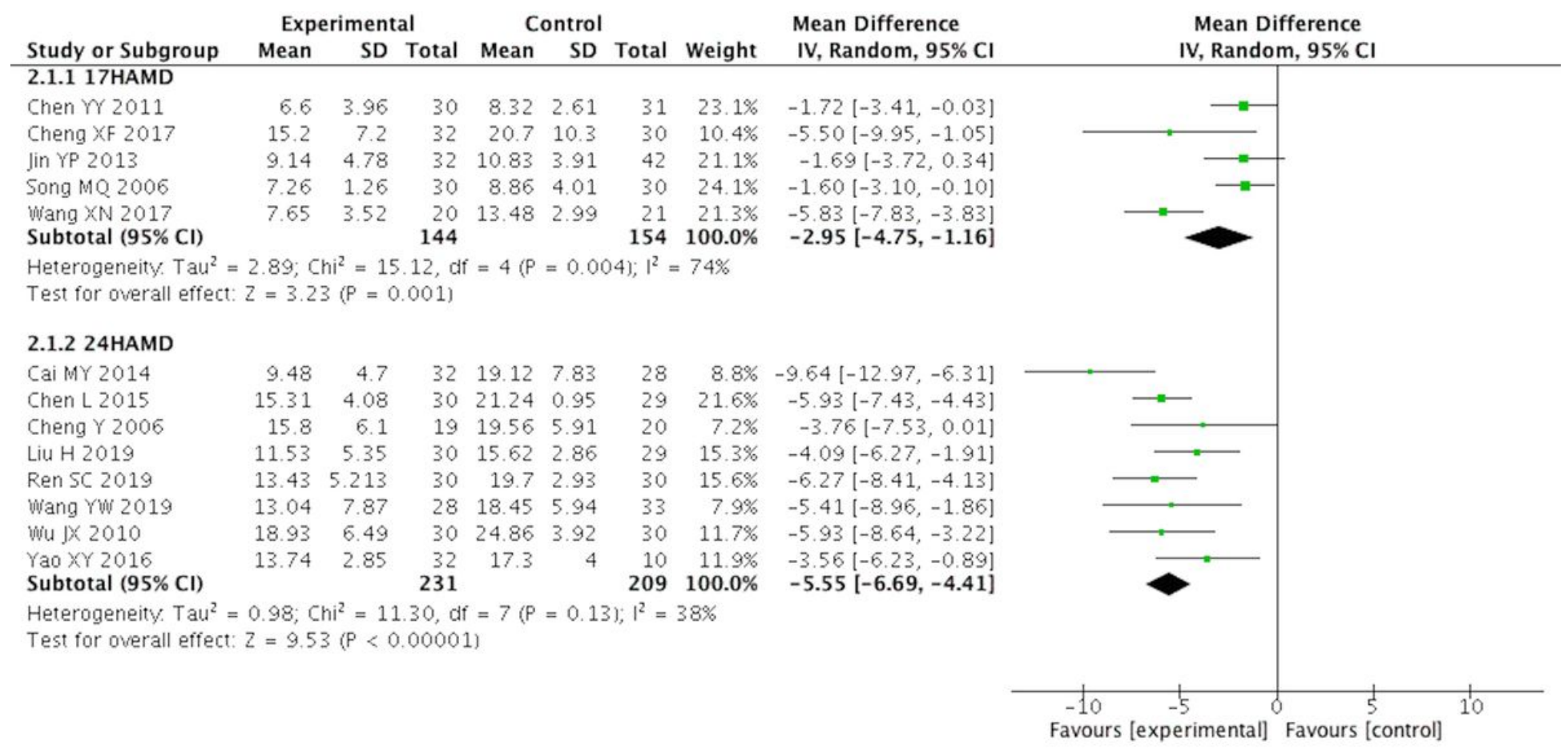

Figure 2

Forest plot of 13-paired included studies. 


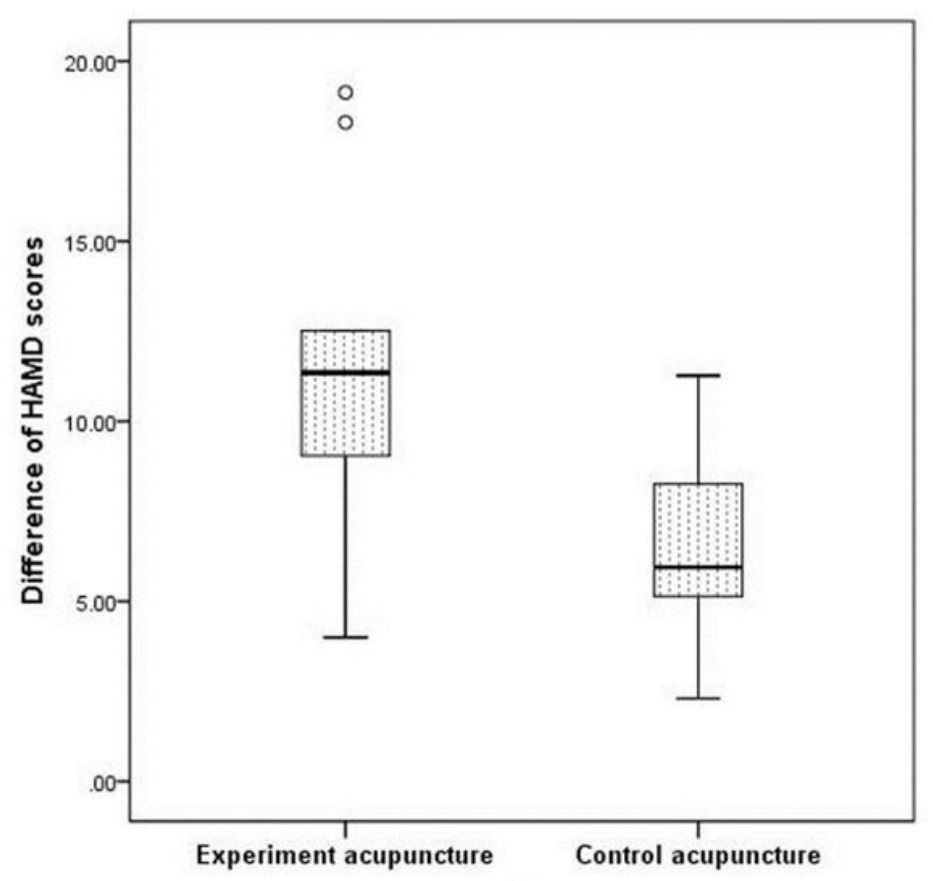

(a)

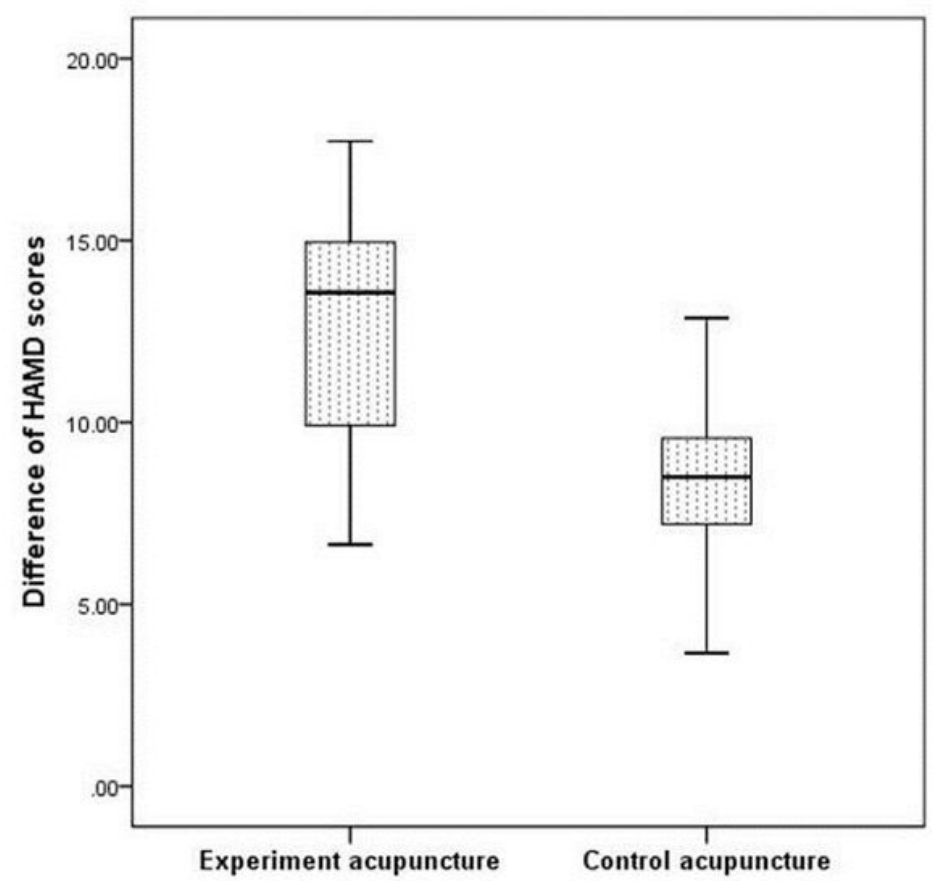

(c)

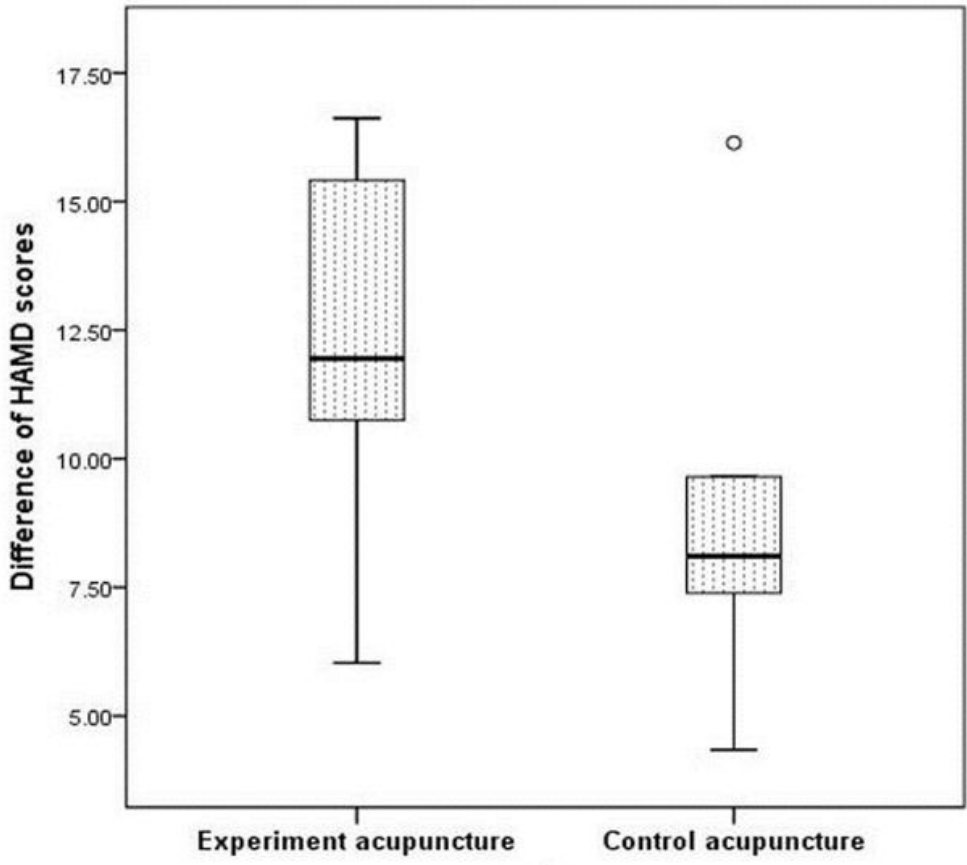

(b)

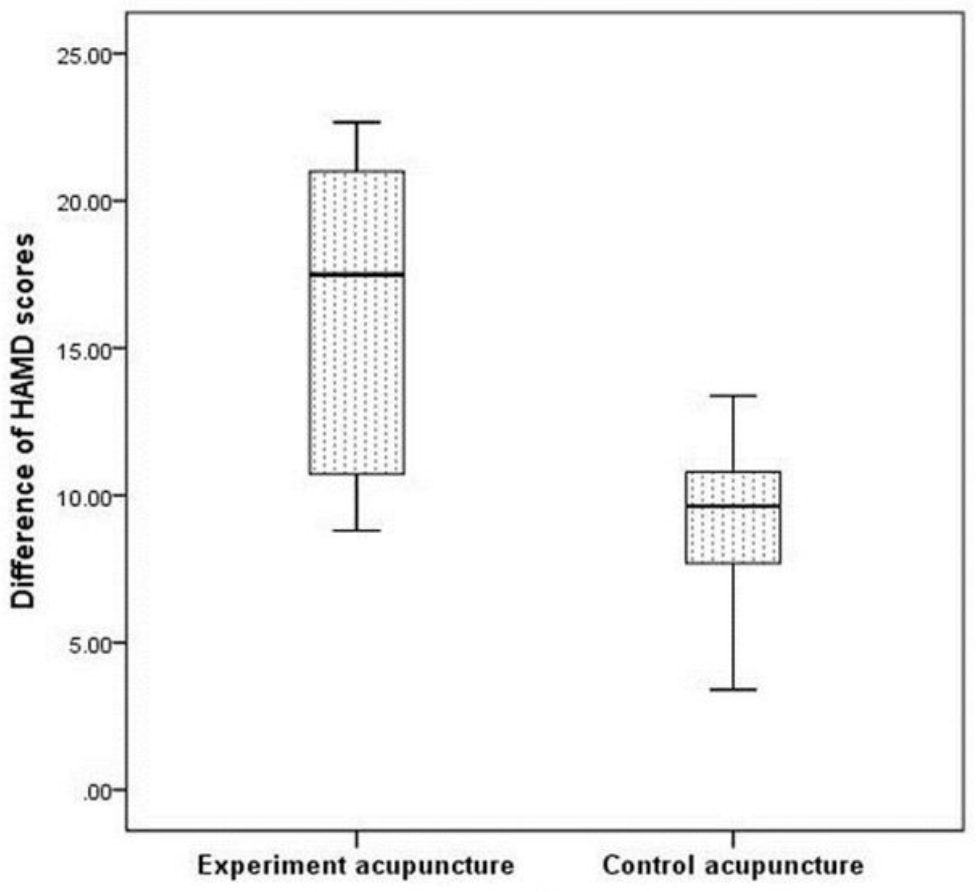

(d)

\section{Figure 3}

Boxplots of the differences between experimental and control acupuncture for Hamilton Depression Scales. (a) Hamilton Depression-17 Scales in four weeks; (b) Hamilton Depression-17 Scales in six weeks; (c) Hamilton Depression-24 Scales in four weeks; (d) Hamilton Depression-24 Scales in six weeks. 